\title{
Glial acetate metabolism is increased following a 72-h fast in metabolically healthy men and correlates with susceptibility to hypoglycemia
}

\author{
David Harry McDougal ${ }^{1}$ (D) Moses Morakortoi Darpolor ${ }^{1} \cdot$ Marina Andreyevna DuVall $^{1}$ - Elizabeth Frost Sutton ${ }^{1,2}$. \\ Christopher David Morrison ${ }^{1} \cdot$ Kishore Murali Gadde $^{1} \cdot$ Leanne Maree Redman $^{1} \cdot$ Owen Thomas Carmichael $^{1}$
}

Received: 28 December 2017 / Accepted: 11 June 2018 / Published online: 22 June 2018

(c) The Author(s) 2018

\begin{abstract}
Aims Prior exposure to insulin-induced hypoglycemia was shown to increase glial acetate metabolism (GAM) during subsequent exposure to hypoglycemia in diabetic individuals. However, it remained unclear whether this effect was dependent on the disease state or the antecedent cause of hypoglycemia. We aimed to establish whether exposure to fasting-induced hypoglycemia was sufficient to produce alterations in GAM in non-diabetic individuals.

Methods GAM was measured via carbon-13 magnetic resonance spectroscopy during infusion of $\left[1-{ }^{13} \mathrm{C}\right]$ acetate before and after a 72-h fast in six metabolically healthy men. All participants were male, aged 18-40 years, with a Body Mass Index of 20.0-27.9 kg/ $\mathrm{m}^{2}$, who consented to reside at Pennington Biomedical Research Center for 4 days. The main outcome measure was the percent enhancement of cerebral $\left[1-{ }^{13} \mathrm{C}\right]$ bicarbonate (the primary metabolic byproduct of glial oxidation of $\left[1-{ }^{13} \mathrm{C}\right]$ acetate). Continuous glucose monitoring was used to measure hypoglycemic episodes during the 72-h fast.

Results As expected, $72 \mathrm{~h}$ of fasting significantly reduced blood glucose levels and resulted in a high frequency of hypoglycemic episodes. Steady-state GAM increased from $53.5 \pm 3.7$ to $61.9 \pm 1.7 \%$ following the 72 -h fast $(p=0.005)$. This increase correlated with greater duration of hypoglycemia experienced during the fast $(r=0.967)$. In addition, subjects with greater GAM at baseline experienced a greater increase in the duration of hypoglycemia experienced during the 72-h fast $(r=0.979)$. Conclusions GAM has potential as a biomarker for susceptibility to hypoglycemic episodes.

Trail registration Clinicaltrials.gov ID: NCT02690168.
\end{abstract}

Keywords Humans $\cdot$ Fasting $\cdot$ Magnetic resonance spectroscopy $\cdot$ Hypoglycemia $\cdot$ Neuroglia $\cdot$ Glucose $\cdot$ Acetates

Managed by Massimo Federici.

Electronic supplementary material The online version of this article (https://doi.org/10.1007/s00592-018-1180-5) contains supplementary material, which is available to authorized users.

David Harry McDougal

David.McDougal@pbrc.edu

1 Pennington Biomedical Research Center, Baton Rouge, LA 70808, USA

2 Present Address: Magee-Womens Research Institute, 204 Craft Ave, Pittsburgh, PA 15213, USA

\section{Introduction}

Hypoglycemia-associated autonomic failure (HAAF) is a serious condition characterized by drastically reduced neuroendocrine responses to hypoglycemia, and the loss of physiological symptoms of hypoglycemia, or hypoglycemia unawareness [1]. The development of HAAF can lead to ever-worsening and often life-threatening episodes of severe hypoglycemia [2]. HAAF is a significant impediment to the maintenance of healthy plasma glucose levels in both type 1 and 2 diabetes [3, 4].

Glial cells are gaining acceptance for their regulatory functions in a variety of neurological conditions [5], including abnormal hypoglycemic counter-regulation [6-11]. Furthermore, neurospectroscopy studies have demonstrated alterations in glial metabolism in persons with diabetes (PWD) [12] and PWD with HAAF [13]. These studies 
assessed glial metabolism using carbon-13 magnetic resonance spectroscopy $\left({ }^{13} \mathrm{C}\right.$ MRS $)$ combined with the infusion of ${ }^{13} \mathrm{C}$ acetate (a monocarboxylate preferentially metabolized by glial cells) $[14,15]$. Using this technique, Mason et al. [12] determined that glial acetate metabolism (GAM) during a hypoglycemic clamp was greater in PWDs than it was in controls. This was reflected by an increase in both the cerebral transport and acetate oxidation in diabetic subjects during hypoglycemia, yet these differences could not be directly attributed to the diabetic state per se. This is because the PWDs in this study displayed significantly reduced plasma levels of counter-regulatory hormones during the hypoglycemic clamp, indicating a high prevalence of HAAF in these subjects.

In a subsequent investigation by Gulanski et al. [13] GAM was assessed in PWDs with and without HAAF during a hypoglycemic clamp. GAM was greater in PWDs with HAAF than in those without HAAF and the non-diabetic control groups [13]. GAM was not different between PWDs without HAAF and controls. The authors suggest that in response to recurrent exposure to low glucose availability, individuals with HAAF may adapt by increasing their utilization of alternative fuels, such as acetate. Together, these results suggest that prior exposure to insulin-induced hypoglycemia in the diabetic disease state is associated with elevated GAM, but that diabetes itself is not sufficient to increase GAM.

Although consensus exists that HAAF is associated with abnormal GAM, it is unclear if hypoglycemia, independent of diabetes, can alter GAM. Furthermore, it is not understood if alterations in GAM are driven by exposure to hypoglycemia directly, as opposed to indirectly through treatment-induced hyperinsulinemia (the underlying antecedent of HAAF). The current study in metabolically healthy male subjects was, therefore, undertaken to understand these issues by using prolonged fasting as an alternative antecedent to hypoglycemia. A 72-h fast is known to produce moderate-to-severe hypoglycemia $[16,17]$ as well as hypoinsulinemia $[16,18,19]$. We hypothesized that in non-diabetic subjects exposed to a 72-h fast, hypoglycemia will induce increased GAM and any observed increase in GAM will be related to the magnitude of the hypoglycemia experienced during the fast.

\section{Materials and methods}

\section{Subjects}

An a priori power analysis (with a mean and standard deviation of 5 and 2.3 , respectively) previously reported in a similar study [13] estimated that a sample size of 6 would provide adequate statistical power $(0.85)$ for our primary outcome variable. Due to known gender differences in the counter-regulatory response to hypoglycemia [18, 20, 21], study enrollment was limited to males $18-40$ years old with a Body Mass Index (BMI) between 20.0 and $27.9 \mathrm{~kg} / \mathrm{m}^{2}$, who were willing to reside on the inpatient unit for 4 days. Subjects were excluded for prior diagnosis of type 1 or 2 diabetes mellitus, fasting glucose $\geq 110 \mathrm{mg} / \mathrm{dL}$, hyperketonuria ( $\geq 15 \mathrm{mg} / \mathrm{dL}$ ), contraindication to MRI, current use of prescription medications, history of or current eating disorder, history of obsessive compulsive disorder, contraindication to prolonged fasting, and consumption of $\geq 10$ alcoholic drinks per week.

\section{Study design}

The study was conducted over 6 months from 11/2016 to $04 / 2017$. A graphical representation of the study design is presented in Figure S1. Recruited subjects were evaluated for eligibility at a single screening visit. Eligible subjects were then scheduled for a 4-day $(\sim 80 \mathrm{~h})$ inpatient stay. Subjects arrived at the inpatient unit in the morning after a 10-h overnight fast (day 0 ) and weight, vital signs and body composition (Lunar iDXA whole-body scanner, General Electric; Milwaukee, WI, USA) were measured. GAM was then measured via ${ }^{13} \mathrm{C}$ MRS, after which subjects were fed and began $\mathrm{a} \approx 75$-h fast when only water could be consumed ad libitum. Continuous glucose monitoring (CGM) was utilized for the duration of the 72-h fast and CGM glucose levels were recorded every $5 \mathrm{~min}$. At $72 \mathrm{~h}$, measurement of GAM was repeated.

\section{Clinical chemistry}

Plasma or serum levels of blood glucose (BG), betahydroxybutyrate (BHB), free fatty acids (FFA), glucagon, insulin, epinephrine and norepinephrine levels were measured by the Pennington Biomedical Clinical Chemistry Core. See supplementary materials for further details of the specific methods used.

\section{GAM}

Glial acetate metabolism was determined via MRS performed on a GE 3T Signa HDxt magnet (GE Healthcare, Milwaukee, WI, USA) with a single loop (8-cm diameter) radiofrequency (rf) coil (Doty Scientific Inc., Columbia, SC, USA). The $8-\mathrm{cm}$ rf coil was tuned and matched to ${ }^{13} \mathrm{C}$ resonance frequency at $3 \mathrm{~T}$, and pulse sequence calibrations were implemented on a $2-\mathrm{mM}$ sodium $\left[1-{ }^{13} \mathrm{C}\right]$ acetate phantom and a $1.76-\mathrm{mM}$ sodium $\left[1-{ }^{13} \mathrm{C}\right]$ bicarbonate phantom. Each 
participant was positioned in a ${ }^{1} \mathrm{H}$-quadrature head coil with the ${ }^{13} \mathrm{C}$ rf coil placed against the participant's cranium. Scout images were acquired to localize and identify occipital lobe of the brain. Specifically, a high-resolution T1-weighted sequence was used to acquire 5-mm-thick slices in the transverse plane. Higher order shimming routine was used to improve the linewidths of spectral resonances. Baseline MRS data was acquired prior to the start of infusion with a free induction decay (FID) chemical shift imaging [22] sequence by implementing the following parameters: timeto-repetition (TR) of $4 \mathrm{~s}$, a flip angle of $75^{\circ}$, a spectral width of $2.2 \mathrm{kHz}, 1024$ sampling points, number of excitation (NEX) of 8, and 296 total number of scans. At the start of $\left[1-{ }^{13} \mathrm{C}\right]$ acetate infusion, the total number of scans parameter was changed to 1808 to acquire a separate dynamic scan over a 2-h time course. For each participant, a 400$\mathrm{mM}$ sodium $\left[1-{ }^{13} \mathrm{C}\right]$ acetate was infused at a rate of $6 \mathrm{mg} / \mathrm{kg}$ (body weight) for the first $5 \mathrm{~min}$ followed by $3 \mathrm{mg} / \mathrm{kg}$ (body weight) for $55 \mathrm{~min}$. Venous blood samples were obtained at 10 -min intervals during the ${ }^{13} \mathrm{C}$ MRS procedures, starting $20 \mathrm{~min}$ prior to $\left[1-{ }^{13} \mathrm{C}\right]$ acetate infusion, and were used for plasma ${ }^{12} \mathrm{C}$ and ${ }^{13} \mathrm{C}$ acetate determination via gas chromatography-mass spectrometry [(GC-MS); (see supplementary material for details of GC-MS methods)] Plasma fraction enrichment of ${ }^{13} \mathrm{C}$ acetate was determined by dividing the plasma concentration of ${ }^{13} \mathrm{C}$ acetate by total plasma acetate concentration $\left\{\left[{ }^{13} \mathrm{C}\right.\right.$ acetate $] /\left(\left[{ }^{13} \mathrm{C}\right.\right.$ acetate $]+\left[{ }^{12} \mathrm{C}\right.$ acetate $\left.\left.]\right)\right\}$.

All spectral data were post-processed in jMRUI version 5.2 [23] and quantitation of metabolite peaks was performed in the time domain with the AMARES (Advanced Method for Accurate, Robust, and Efficient Spectral fitting) algorithm [24]. FID data were apodized with $2 \mathrm{~Hz}$, fast Fourier transformed, and manually phased (zero-order phase). Confirmed bicarbonate resonance peak data was transferred into Matlab (MathWorks, Natick, MA, USA), normalized with respect to the total sum and fitted to a mono-exponential equation as previously described by Bluml et al. [25] (see supplementary materials for details of equations).

\section{CGM}

A G4 Platinum CGM system (Dexcom, San Diego, CA, USA) was used per manufacturer's instructions to measure interstitial glucose. The sensor was calibrated every $12 \mathrm{~h}$ via capillary BG measurements by finger stick using a HemoCue B-glucose analyzer (HemoCue, Brea, CA, USA). After completing the fast, CGM data was exported to Microsoft Excel to determine the mean, maximum, and minimum BG, as well as percent time spent $\leq 70 \mathrm{mg} / \mathrm{dL}$ and frequency of hypoglycemic episodes. A hypoglycemic episode was defined as three or more consecutive CGM glucose readings $\leq 70 \mathrm{mg}$ / $\mathrm{dL}$ (Level 1) or $\leq 54 \mathrm{mg} / \mathrm{dL}$ (Level 2) [26].

\section{Statistics}

Results are reported as mean \pm SE. All statistical analyses were performed using SAS Version 9.4 (SAS Institute, Cary, NC, USA) via a linear mixed effect model for repeated measures. The mixed effect model allowed all subjects to be included in the analyses irrespective of missing MRS data (see "GAM" for details) and models the correlation between the same subjects using a compound symmetric covariance matrix. Two sample $t$ tests, based on estimates from the mixed model, were used to determine differences between day 0 and day 3 levels of plasma levels of metabolites and hormones, steady-state $\mathrm{PE}$ of ${ }^{13} \mathrm{C}$ bicarbonate, and metabolic modeling parameters. Distributional assumptions of the residuals from these models were investigated using both visual inspection as well as Shapiro-Wilks tests. Pearson's correlation was used to determine the association between time $\leq 70$ and steady-state PE of bicarbonate on days 0 and 3. For all statistical analyses, $p$ values of $<0.05$ were considered statistically significant.

\section{Results}

Baseline characteristics and demographics of the subjects are presented in Table 1. Subjects experienced a statistically significant weight loss $(3.7 \pm 0.4 \%)$ following the 72 -h fast. All subjects had normoglycemia $(85.1 \pm 2.8 \mathrm{mg} / \mathrm{dL})$ on day 0 (following a 12-h fast). Blood glucose levels were significantly reduced on day 3 (following the 72-h fast), exhibiting a $16 \%$ decrease relative to values obtained on day 0 . Serum concentrations of FFA, glucagon, and epinephrine were significantly increased on day 3 (Table 2). Serum norepinephrine and acetate levels, however, were not changed (Table 2). There was no evidence of hyperinsulinemia on day 0 and insulin significantly decreased in response to the 72-h fast with concentrations below the level of detection $(2.0 \mu \mathrm{U} / \mathrm{mL})$ for $5 / 6$ subjects. Similarly, there was no evidence of ketosis on day 0 ; however, the 72-h fast significantly increased BHB measured in serum and urine (Table 2).

$\left[1-{ }^{13} \mathrm{C}\right]$ acetate infusion produced a rapid increase in the plasma fractional enrichment of $\left[1-{ }^{13} \mathrm{C}\right]$ acetate within $10 \mathrm{~min}$. Mean plasma fractional enrichment of $\left[1-{ }^{13} \mathrm{C}\right]$ acetate did not differ between day 0 and day $3(70.32 \pm 0.02$ and $69.26 \pm 0.02 \%$, respectively; $p=0.61$ ), nor did plasma levels of $\left[1-{ }^{13} \mathrm{C}\right]$ acetate (data not shown). Prior to the infusion of $\left[1-{ }^{13} \mathrm{C}\right]$ acetate, no spectral peaks associated with acetate metabolism were detected (Figure S2, panel A). With the onset of the $\left[1-{ }^{13} \mathrm{C}\right]$ acetate infusion, a rapid increase in the cerebral PE of ${ }^{13} \mathrm{C}$ bicarbonate was observed (Fig. 1a). This enhancement reflects the glial oxidation of our infusate via the TCA cycle. Additional peaks associated with $\left[1-{ }^{13} \mathrm{C}\right]$ acetate oxidation by glial cells, including $\left[1-{ }^{13} \mathrm{C}\right]$ glutamine, 
Table 1 Subjects demographics and characteristics

\begin{tabular}{ll}
\hline & Subjects \\
\hline Sex & \\
Male, $n(\%)$ & $6(100)$ \\
Race & \\
Black or African-American $n,(\%)$ & $3(50)$ \\
White $n,(\%)$ & $3(50)$ \\
Age & $28.7 \pm 1.0$ \\
Weight $(\mathrm{kg})$ & $76.1 \pm 5.6$ \\
BMI (at screening) & $23.5 \pm 1.1$ \\
Fasting glucose (mg/dL) & $84.8 \pm 3.7$ \\
Body fat by iDXA, $\%$ & $17.6 \pm 3.0$ \\
Weight loss after the 72-h fast $(\%)$ & $3.7 \pm 0.4$ \\
\hline
\end{tabular}

Data are $n(\%)$ or mean \pm SE; BMI was calculated based on screening anthropometrics; weight and body fat reflect day 0 measurements

$S E$ standard error, BMI Body Mass Index

Table 2 Levels of metabolites and hormones on day 0 and day 3, following a 12-h fast and 72-h fast, respectively

\begin{tabular}{llll}
\hline & $\begin{array}{l}\text { Day 0 } \\
\text { 12-h fast }\end{array}$ & $\begin{array}{l}\text { Day 3 } \\
72 \text {-h fast }\end{array}$ & $p$ value \\
\hline Glucose, mg/dL & $85.1 \pm 2.8$ & $71 \pm 2.0^{*}$ & 0.005 \\
Free fatty acid, mmol/L & $0.6 \pm 0.1$ & $1.1 \pm 0.2^{*}$ & 0.015 \\
Serum $\beta$-hydroxybutyrate, $\mathrm{mmol} / \mathrm{L}$ & $0.1 \pm 0.0$ & $2.3 \pm 0.5^{*}$ & 0.008 \\
Urine $\beta$-hydroxybutyrate, mmol/L & - & $20 \pm 13$ & \\
Glucagon, pg/mL & $56 \pm 5.2$ & $88 \pm 7.0^{*}$ & 0.012 \\
Insulin, $\mu \mathrm{U} / \mathrm{mL}$ & $3.1 \pm 1.0$ & - & \\
Epinephrine, $\mathrm{ng} / \mathrm{mL}$ & $50 \pm 6.7$ & $72 \pm 4.8^{*}$ & 0.041 \\
Norepinephrine, ng/mL & $380 \pm 63$ & $540 \pm 110$ & 0.108 \\
Acetate $(12-\mathrm{C}), \mu \mathrm{mol} / \mathrm{L}$ & $152 \pm 6.1$ & $167 \pm 20$ & 0.106 \\
\hline
\end{tabular}

Data are mean \pm SE. Data omitted when values were below minimal detection limits of the assay

$S E$ standard error

*Significant difference between 12- and 72-h values as determined by paired $t$ tests, $p$ value $<0.05$

$\left[5-{ }^{13} \mathrm{C}\right]$ glutamine, $\left[1-{ }^{13} \mathrm{C}\right]$ glutamate, and $\left[5-{ }^{13} \mathrm{C}\right]$ glutamate, were also observed in our spectra (Figures S2B and S2C). Total PE of ${ }^{13} \mathrm{C}$ bicarbonate approached steady-state within 60-70 min (Fig. 1a) and persisted in our spectra following the offset of the $\left[1-{ }^{13} \mathrm{C}\right]$ acetate infusion (Figures S2B and $\mathrm{S} 2 \mathrm{C})$. Figure $1 \mathrm{~b}$ displays the average curve-fit for all days 0 and 3 scans, respectively, at approximately 15 -min intervals. A clear increase in the $\mathrm{PE}$ of ${ }^{13} \mathrm{C}$ bicarbonate is shown in the average day 3 curve-fit relative to day 0 , with a statistically significant increase in PE of bicarbonate on day 3 versus day 0 curve fits $(p<0.0001)$. The curve-fitting procedure also yielded the cerebral metabolic rate constants $\mathrm{CMR}_{\mathrm{ACE}}$, $\mathrm{V}_{\mathrm{TCA}}, \mathrm{V}_{\mathrm{ACE}}$, and $\mathrm{CO}_{2}$ from Eq. (2) as described in supplimentary materials. There were no significant differences in
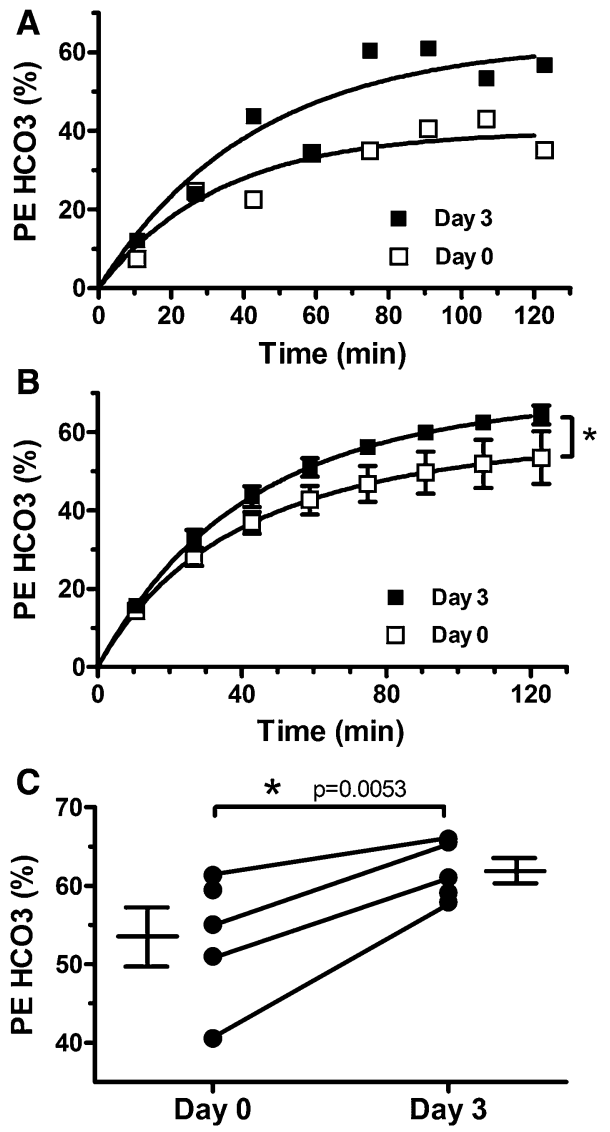

Fig. 1 Glial acetate metabolism (GAM) is increased following a 72-h fast. GAM was measured via carbon 13 magnetic resonance spectroscopy during $\left[1-{ }^{13} \mathrm{C}\right]$ acetate infusion. a Time course of the percent enhancement (PE) of ${ }^{13} \mathrm{C}$ bicarbonate $\left(\mathrm{HCO}_{3}\right.$; the primary metabolite of $\left[1-{ }^{13} \mathrm{C}\right]$ acetate oxidation) following the start of infusion in a representative subject after $12 \mathrm{~h}$ of fasting (day 0 ) and $72 \mathrm{~h}$ of fasting (day 3 ). The solid line represents the best fit of a mono-exponential function modeling GAM. b Comparison of the average best fit across subjects via a linear mixed effects model demonstrates a clear increase from day 0 to $3(p<0.0001)$. c Average steady-state $\mathrm{PE}$ of $\mathrm{HCO}_{3}$ was increased on day 3 relative to day $0(p=0.0053)$. Furthermore, the PE of $\mathrm{HCO} 3$ was increased on day 3 relative to day 0 in all subjects who completed both scans (symbols with connecting lines)

the average values of these rate constants for day 0 versus day 3 (Table S1). Mean steady-state PE of ${ }^{13} \mathrm{C}$ bicarbonate measured from 64 to 96 min following the initiation of the $\left[1-{ }^{13} \mathrm{C}\right]$ acetate infusion also increased on day 3 relative to day $0(61.9 \pm 1.7$ versus $53.5 \pm 3.7 ; p=0.005)$. The day 0 values of PE of bicarbonate displayed increased inter-subject variability relative to day 3 values. Day 0 values ranged from 40.6 to $61.4 \%$, while day 3 values ranged from 57.9 to $66.0 \%$ (Fig. 1c). Furthermore, in the 4 subjects with complete data on day 0 and day 3 , PE of ${ }^{13} \mathrm{C}$ bicarbonate was increased on day 3 relative to day 0 (filled circles with connecting lines in Fig. 1c). Altogether, these data reflect an increase in the rate of glial metabolism of $\left[1-{ }^{13} \mathrm{C}\right]$ acetate on day 3 relative to day 0 . 
CGM glucose values were not significantly different from BG values measured at identical time points $(p=0.1835$; data not shown). CGM glucose levels dropped well below $80 \mathrm{mg} / \mathrm{dL}$ during the first $24 \mathrm{~h}$ of the fast. On day 2, glucose levels rebounded to approximately $90 \mathrm{mg} / \mathrm{dL}$ before falling again toward hypoglycemic levels $(<70 \mathrm{mg} / \mathrm{dL})$ during the last $24 \mathrm{~h}$ (Fig. 2, top panel). The subjects' mean CGM glucose level during the 72-h fast was well within the normal range $(70-150 \mathrm{mg} / \mathrm{dL})$. However, the mean minimum CGM reading was below $70 \mathrm{mg} / \mathrm{dL}$ (Table 3), and the subjects experienced considerable time below $70 \mathrm{mg} / \mathrm{dL}$ (Fig. 2). The number of subjects who experienced at least one episode of Level 1 hypoglycemia was five (83.3\%), four $(60 \%)$, and five $(83.3 \%)$ during the first, second, and third days of fasting, respectively. The number of subjects who experienced at least one episode of Level 2 hypoglycemia was two $(33.3 \%)$, zero $(0 \%)$, and one $(16.7 \%)$ during the first, second, and third days of fasting, respectively. The percent time spent in the hypoglycemic state $(<70 \mathrm{mg} / \mathrm{dL})$ was positively associated with the PE of bicarbonate measured on day 0 (Pearson's $r^{2}=0.96, p=0.004$; Fig. 3a). Furthermore, an increased exposure to hypoglycemia was positively associated with increased GAM at the end of the 72-h fast (Pearson's $r^{2}=0.94, p=0.007$; Fig. 3b).
Table 3 Continuous glucose monitoring parameters during the 72-h fast

$\begin{array}{lr}\text { Mean blood glucose, mg/dL } & 84 \pm 4.3 \\ \text { Maximum, mg/dL } & 118 \pm 7.5 \\ \text { Minimum, mg/dL } & 51 \pm 5.7 \\ \text { Time at }<70 \mathrm{mg} / \mathrm{dL}, \% & 18 \pm 5.8 \\ \text { Time at }<54 \mathrm{mg} / \mathrm{dL}, \% & 3.5 \pm 1.8\end{array}$

Data are mean $\pm \mathrm{SE}$ or $\%$

$S E$ standard error

\section{Discussion}

Alterations in GAM are associated with a variety of clinical conditions in humans [27] including abnormal hypoglycemic counter-regulation $[12,13]$. In this study, we used ${ }^{13} \mathrm{C}$ MRS in non-diabetic subjects to assess whether GAM increased following a 72-h fast (a natural initiator of hypoglycemia). The major findings of this study were: (1) a 72-h fast in metabolically healthy men increases GAM, (2) increased exposure to hypoglycemia during the fast is associated with larger increases in GAM, and (3) increased GAM measured prior to the 72-h fast is correlated with increased susceptibility to hypoglycemia during the fast. The first two findings support our original hypothesis, while the third finding
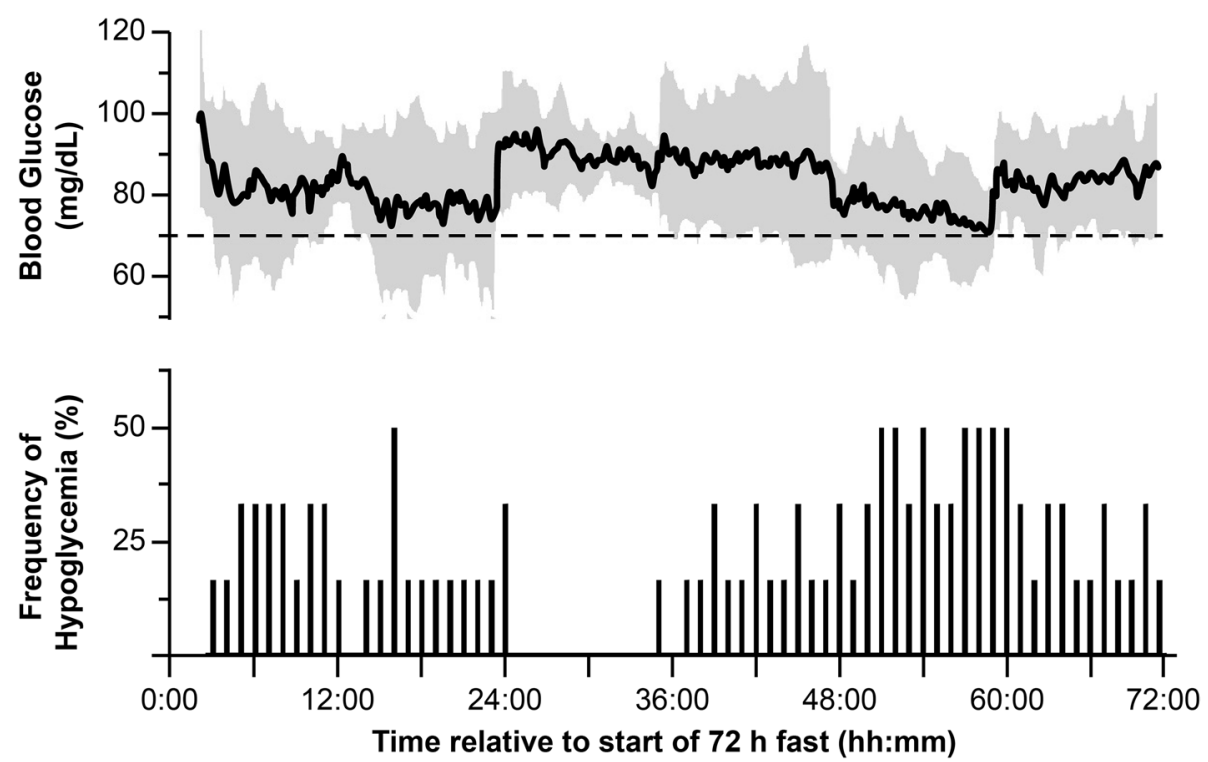

Fig. 2 Continuous glucose monitoring (CGM) demonstrates that $72 \mathrm{~h}$ of fasting leads to frequent bouts of hypoglycemia. CGM was used to monitor blood glucose levels at 5-min intervals throughout a 72-h fast. The mean blood glucose for all six subjects is plotted in the top panel. The gray shading represents the $95 \%$ confidence limit and the dotted line represents the clinical threshold for Level 1 hypoglycemia,
$70 \mathrm{mg} / \mathrm{dL}$. The lower panel represents the percent of subjects which experienced a hypoglycemic episode during each hour of the 72-h fast. A subject was determined to have had a hypoglycemic episode during a given hour of the fast if a CGM reading of $\leq 70 \mathrm{mg} / \mathrm{dL}$ was maintained for three consecutive CGM readings (15 min) 

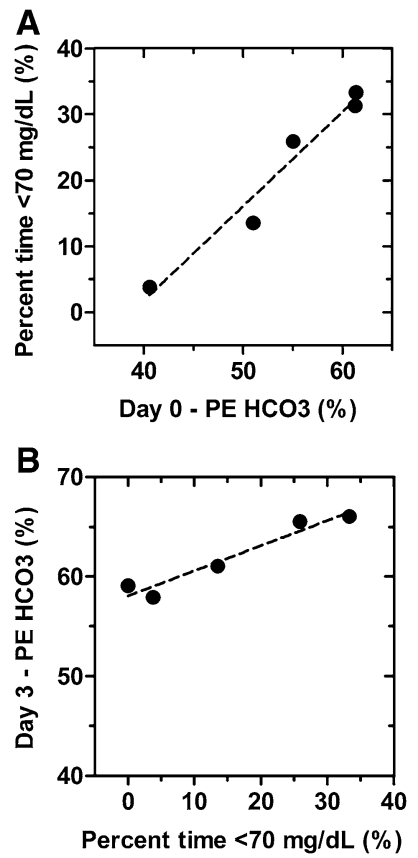

Fig. 3 Individual differences in glial acetate metabolism (GAM) are related to the duration of hypoglycemia experienced during a 72-h fast. GAM, as measured by the percent enhancement of ${ }^{13} \mathrm{C}$ bicarbonate $\left(\mathrm{PE}\right.$ of $\mathrm{HCO}_{3}$ ) following an acetate infusion, is both predictive of and responsive to subsequent fasting-induced hypoglycemia. a Relationship between glia acetate metabolism measured just prior to $72 \mathrm{~h}$ of fasting (day 0 ) and the subsequent exposure to hypoglycemia during the fast (percent time $\leq 70 \mathrm{mg} / \mathrm{dL}$ ). A correlation analysis between these two variables demonstrated a robust positive relationship between GAM and the duration of hypoglycemia experienced during fasting (Pearson's $r^{2}=0.96, p=0.004$ ). b GAM measured following a 72-h fast (day 3 ) increase in a dose-dependent manner based on the magnitude of exposure to hypoglycemia during the fast (Pearson's $r^{2}=0.94, p=0.007$ )

was unexpected and suggests that ${ }^{13} \mathrm{C}$ MRS is a promising marker for hypoglycemia risk.

Hypoglycemia is a major complication of type 1 and 2 diabetes. Recurrent exposure to insulin-induced hypoglycemia can lead to the development of HAAF (a lifethreatening metabolic disorder associated with blunted hypoglycemic counter-regulation). Previous studies demonstrated that HAAF is associated with increased GAM in PWDs during acute hypoglycemia $[12,13]$. Our results advance these finding by demonstrating that exposure to hypoglycemia alone (independent of diabetes or exposure to hyperinsulinemia) is sufficient to increase glial metabolism. Furthermore, using continuous glucose monitoring to document the magnitude of hypoglycemic exposure, we demonstrated for the first time that exposure to hypoglycemia leads to a dose-dependent increase in GAM.

Our observation that GAM may determine susceptibility to fasting-induced hypoglycemia is also noteworthy in light of previous work. Gulanski et al. [13] showed that during a hypoglycemic clamp, GAM in both PWDs and non-diabetic subjects was inversely related to their neuroendocrine responses to the clamp. Thus, subjects with increased GAM displayed reduced hypoglycemic counterregulation, thereby demonstrating increased susceptibility to hypoglycemia. In addition, our finding regarding to the metabolic rate constants associated cerebral acetate metabolism (Table S1) are consistent with those of Gulanski et al. [13], with the exception that Gulanski et al. [13] did observe a $50 \%$ increase in $\mathrm{CMR}_{\mathrm{ACE}}$. Altogether, our findings along with those of Gulanski et al. [13] suggest that increased GAM is a predictor of increased susceptibility to hypoglycemia independent of disease state, acute glycemic condition (hypoglycemic versus euglycemic), and the cause of the hypoglycemia (fasting or hyperinsuline$\mathrm{mia}$ ). These data suggest that further investigations into the relationship between GAM and HAAF may provide key mechanistic and diagnostic insights which could lead to better treatment outcomes for PWD.

The clinical management of HAAF is severely hampered by the lack of non-invasive, objective biomarkers useful for definitive diagnosis and for ascertaining the severity of HAAF in PWD. Although our study was not performed in PWDs, our results are applicable, since the physiological response to fasting-induced hypoglycemia is identical to that of insulin-induced hypoglycemia [28], and exposure to fasting-induced hypoglycemia produces HAAF-like symptoms in humans [29, 30]. Our observation that GAM during euglycemia may predict subsequent susceptibility to hypoglycemia raises the possibility for targeted assessment of GAM to assess hypoglycemic risk in PWD.

Several limitations of our study are worth noting. Our study was adequately powered to detect our primary endpoint, change in PE of bicarbonate. Yet, the robustness of correlations between the PE of bicarbonate and hypoglycemia, as well as our CGM dataset, would be strengthened by an increased sample size. Although CGM is commonly used to clinically monitor hypoglycemia, CGM glucose levels are not as accurate as blood glucose measurements. This limitation was offset somewhat by our choice of the Dexcom G4 device, which has been shown to be significantly more accurate than comparable devices [31]. In addition, our use of $\left[1-{ }^{13} \mathrm{C}\right]$ acetate instead $\left[2-{ }^{13} \mathrm{C}\right]$ acetate precluded determination of brain ${ }^{13} \mathrm{C}$ acetate concentrations due to the close spectral proximity of $1-{ }^{13} \mathrm{C}$ acetate and $5{ }^{13} \mathrm{C}$ glutamine, a downstream metabolite of $1-{ }^{13} \mathrm{C}$ acetate oxidation (Figure S2B). 


\section{Conclusion}

Using a unique natural paradigm to induce hypoglycemia in healthy men, and a sophisticated ${ }^{13} \mathrm{C}$ MRS protocol, our study significantly advances the scope and importance of the relationship between GAM and hypoglycemia. Increases in GAM are directly proportional to the extent of hypoglycemia exposure, in a dose-dependent manner, and higher levels of GAM under euglycemic conditions are associated with susceptibility to hypoglycemia. These two findings are very important clinically, i.e., GAM is possibly a novel biomarker of hypoglycemic risk and its measurement by ${ }^{13} \mathrm{C}$ MRS has the potential to provide a much-needed diagnostic tool for the detection and treatment of HAAF.

Acknowledgements This work was supported in part by 1 U54 GM104940 from the National Institute of General Medical Sciences of the National Institutes of Health, which funds the Louisiana Clinical and Translational Science Center and P30 DK072476, the Louisiana Nutrition Obesity Research Center. This work was also supported by the Pennington Biomedical Research Foundation. The content is solely the responsibility of the authors and does not necessarily represent the official views of the National Institutes of Health. The authors would like to thank the staff of the PBRC Inpatient and Outpatient clinics for their invaluable support of this study, as well as Dr. Robbie Beyl for providing statistical analyses and consultation.

\section{Compliance with ethical standards}

Conflict of interest The authors have stated explicitly that there are no conflicts of interest in connection with this article.

Ethical standard statement All study procedures and literature were approved by the Institutional Review Board at Pennington Biomedical Research Center (PBRC). All subjects provided informed consent prior to enrollment in the study (NCT02690168). All study procedures and measurements, except plasma acetate assays (conducted at the University of Tennessee, Knoxville, TN, USA), were performed at PBRC, Baton Rouge, LA, USA.

Informed consent Informed consent was obtained from all individual participants in the study.

Open Access This article is distributed under the terms of the Creative Commons Attribution 4.0 International License (http://creativeco mmons.org/licenses/by/4.0/), which permits unrestricted use, distribution, and reproduction in any medium, provided you give appropriate credit to the original author(s) and the source, provide a link to the Creative Commons license, and indicate if changes were made.

\section{References}

1. Ritter S (2017) Monitoring and maintenance of brain glucose supply: importance of hindbrain catecholamine neurons in this multifaceted task. In: harris rbs (ed) appetite and food intake: central control, 2nd edn. CRC Press/Taylor \& Francis, Boca Raton, pp 177-204

2. Cryer PE (2013) Mechanisms of hypoglycemia-associated autonomic failure in diabetes. N Engl J Med 369:362-372

3. Cryer PE (2008) The barrier of hypoglycemia in diabetes. Diabetes 57:3169-3176

4. Segel SA, Paramore DS, Cryer PE (2002) Hypoglycemia-associated autonomic failure in advanced type 2 diabetes. Diabetes 51:724-733

5. Miller DW, Cookson MR, Dickson DW (2004) Glial cell inclusions and the pathogenesis of neurodegenerative diseases. Neuron Glia Biol 1:13-21

6. Sanders NM, Dunn-Meynell AA et al (2004) Third ventricular alloxan reversibly impairs glucose counterregulatory responses. Diabetes 53:1230-1236

7. Marty N, Dallaporta M, Foretz M et al (2005) Regulation of glucagon secretion by glucose transporter type 2 (glut2) and astrocytedependent glucose sensors. J Clin Investig 115:3545-3553

8. Hermann GE, Viard E, Rogers RC (2014) Hindbrain glucoprivation effects on gastric vagal reflex circuits and gastric motility in the rat are suppressed by the astrocyte inhibitor fluorocitrate. J Neurosci 34:10488-10496

9. McDougal DH, Viard E, Hermann GE et al (2013) Astrocytes in the hindbrain detect glucoprivation and regulate gastric motility. Auton Neurosci 175:61-69

10. Rogers RC, Ritter S, Hermann GE (2016) Hindbrain cytoglucopenia-induced increases in systemic blood glucose levels by 2-deoxyglucose depend on intact astrocytes and adenosine release. Am J Physiol Regul Integr Comp Physiol 310:R1102-R1108

11. Rogers RC, McDougal DH, Hermann GE (2017) Hindbrain astrocyte glucodetectors and counterregulation. In: Harris RBS (ed) Appetite and food intake: central control, 2nd edn. CRC Press/ Taylor \& Francis, Boca Raton, pp 205-228

12. Mason GF, Petersen KF, Lebon V et al (2006) Increased brain monocarboxylic acid transport and utilization in type 1 diabetes. Diabetes 55:929-934

13. Gulanski BI, De Feyter HM, Page KA et al (2013) Increased brain transport and metabolism of acetate in hypoglycemia unawareness. J Clin Endocrinol Metab 98:3811-3820

14. Deelchand DK, Shestov AA, Koski DM et al (2009) Acetate transport and utilization in the rat brain. J Neurochem 109(Suppl 1):46-54

15. Fonnum F, Johnsen A, Hassel B (1997) Use of fluorocitrate and fluoroacetate in the study of brain metabolism. Glia 21:106-113

16. Bak AM, Møller AB, Vendelbo MH et al (2016) Differential regulation of lipid and protein metabolism in obese vs. lean subjects before and after a 72-h fast. Am J Physiol Endocrinol Metab 311:E224-E235

17. Ding XQ, Maudsley AA, Schweiger U et al (2017) Effects of a 72 hours fasting on brain metabolism in healthy women studied in vivo with magnetic resonance spectroscopic imaging. J Cereb Blood Flow Metab 38:469-478 (271678X17697721)

18. Hojlund K, Wildner-Christensen M, Eshoj O et al (2001) Reference intervals for glucose, beta-cell polypeptides, and counterregulatory factors during prolonged fasting. Am J Physiol Endocrinol Metab 280:E50-E58

19. Vella A, Service FJ, O’Brien PC (2003) Glucose counterregulatory hormones in the 72-hour fast. Endocr Pract 9:115-118

20. Diamond MP, Jones T, Caprio S et al (1993) Gender influences counterregulatory hormone responses to hypoglycemia. Metab Clin Exp 42:1568-1572

21. Amiel SA, Maran A, Powrie JK et al (1993) Gender differences in counterregulation to hypoglycaemia. Diabetologia 36:460-464

22. Skamarauskas JT, Oakley F, Smith FE et al (2014) Noninvasive in vivo magnetic resonance measures of glutathione synthesis in 
human and rat liver as an oxidative stress biomarker. Hepatology 59:2321-2330

23. Vanhamme L, van den Boogaart A, Van Huffel S (1997) Improved method for accurate and efficient quantification of MRS data with use of prior knowledge. J Magn Reson 129:35-43

24. Stefan D, Cesare FD, Andrasescu A et al (2009) Quantitation of magnetic resonance spectroscopy signals: the jMRUI software package. Meas Sci Technol 20:104035

25. Bluml S, Moreno-Torres A, Shic F et al (2002) Tricarboxylic acid cycle of glia in the in vivo human brain. NMR Biomed 15:1-5

26. International Hypoglycaemia Study Group (2017) Glucose concentrations of less than $3.0 \mathrm{mmol} / \mathrm{L}(54 \mathrm{mg} / \mathrm{dL})$ should be reported in clinical trials: a joint position statement of the American Diabetes Association and the European Association for the Study of Diabetes. Diabetes Care 40:155-157

27. Ross B, Lin A, Harris $\mathrm{K}$ et al (2003) Clinical experience with ${ }^{13} \mathrm{C}$ MRS in vivo. NMR Biomed 16:358-369
28. Boyle PJ, Shah SD, Cryer PE (1989) Insulin, glucagon, and catecholamines in prevention of hypoglycemia during fasting. Am J Physiol 256:E651-E661

29. Adamson U, Lins PE, Grill V (1989) Fasting for $72 \mathrm{~h}$ decreases the responses of counterregulatory hormones to insulin-induced hypoglycaemia in normal man. Scand J Clin Lab Investig 49:751-756

30. Drenick EJ, Alvarez LC, Tamasi GC et al (1972) Resistance to symptomatic insulin reactions after fasting. J Clin Investig 51:2757-2762

31. Kropff J, Bruttomesso D, Doll W et al (2015) Accuracy of two continuous glucose monitoring systems: a head-to-head comparison under clinical research centre and daily life conditions. Diabetes Obes Metab 17:343-349 